\title{
Ecological stability of broad bean (Vicia faba L.) in organic farming conditions
}

\author{
N.A. Georgieva $\otimes$, V.I. Kosev \\ Institute of Forage Crops, Pleven, Bulgaria \\ 凶e-mail: imnatalia@abv.bg
}

\begin{abstract}
The main direction in the breeding of legumes is the development of high-productive cultivars with stable yields. In order to assess the ecological stability of 17 broad bean accessions (Fb 1896, Fb 1903, Fb 1929, Fb 2481, Fb 2486, Fb 3270, BGE 002106, BGE 029055, BGE 032012, BGE 041470, BGE 043776, BGE 046721, FbH 13, FbH 14, FbH 15, $\mathrm{FbH} 16, \mathrm{BGP}$ ) with regard to key quantitative traits, a field experiment was conducted in the Institute of Forage Crops (Pleven) in 2016-2018. Plants were grown under organic farming conditions without the use of fertilizers or pesticides. Three types of stability parameters were calculated based on regression, variance, and nonparametric analysis. The results of the variance analysis showed a significant genotype x environment interaction for all quantitative traits except pod width. The factor 'environment' had the greatest impact on the phenotypic manifestation of the traits, followed by the factors 'genotype' and 'genotype xenvironment interaction'. In terms of plant height and $1 \mathrm{st}$ pod height, accessions $\mathrm{FbH} 16$ and $\mathrm{FbH} 13$ can be classified as high $(79 \mathrm{~cm}, 35 \mathrm{~cm})$ and ecologically stable (bi $=0.76$, bi $=0.79$ ). BGE 029055 was little variable and had high numbers of pods (15) and seeds (41) per plant. Accessions $\mathrm{FbH} 14, \mathrm{FbH} 16, \mathrm{FbH} 15$, and BGP were distinguished by high seed weight per plant (from 28.36 to $34.93 \mathrm{~g}$ ), but they exhibited instability ( $\mathrm{bi}>1$ ) under unfavorable environmental conditions. In contrast, Fb 1903, BGE 043776, and Fb 3270 were very stable (bi<1) but low-productive. Accessions Fb 1896, Fb 1929, Fb 2481, $\mathrm{Fb}$ 2486, BGE 002106, and BGE 029055 showed intermediate parameters, as they had the coefficient of linear regression close to 1, but they were also low-productive. BGE 041470 appeared to be of special interest for breeding. It had high values of 100 seed mass $(101.38 \mathrm{~g})$ and seed weight per plant $(32.14 \mathrm{~g})$, being relatively stable $(\mathrm{bi}=1.10)$. GGE biplot analysis determined accessions BGE 046721, BGE 032012, FbH 15 and FbH 16 as a promising breeding material combining high and stable seed yield.
\end{abstract}

Key words: Vicia faba L.; stability parameter; productivity; traits; accessions; regression analysis; nonparametric analysis.

For citation: Georgieva N.A., Kosev V.I. Ecological stability of broad bean (Vicia faba L.) in organic farming conditions. Vavilovskii Zhurnal Genetiki i Selektsii =Vavilov Journal of Genetics and Breeding. 2019;23(8):981-992. DOI 10.18699/VJ19.36-o

\section{Экологическая стабильность боба овощного (Vicia faba L.) в условиях органического хозяйства}

\author{
Н.А. Георгиева 凶, В.И. Косев \\ Институт кормовых культур, Плевен, Болгария \\ बe-mail: imnatalia@abv.bg
}

Важное направление в селекции бобовых - создание высокоурожайных сортов со стабильной продуктивностью. Для оценки экологической стабильности 17 образцов Vicia faba (Fb 1896, Fb 1903, Fb 1929, Fb 2481, Fb 2486, Fb 3270, BGE 002106, BGE 029055, BGE 032012, BGE 041470, BGE 043776, BGE 046721, FbH 13, FbH 14, $\mathrm{FbH} \mathrm{15,} \mathrm{FbH} \mathrm{16,} \mathrm{BGP)} \mathrm{по} \mathrm{основным} \mathrm{количественным} \mathrm{признакам} \mathrm{в} \mathrm{Институте} \mathrm{кормовых} \mathrm{культур} \mathrm{(г.} \mathrm{Плевен,} \mathrm{Бол-}$ гария) в 2016-2018 гг. был проведен полевой эксперимент. Растения выращивали в условиях органического земледелия, без использования удобрений и пестицидов. На основе регрессионного, дисперсионного и непараметрического анализа были рассчитаны три типа параметров стабильности. Результаты дисперсионного анализа доказывают взаимодействие генотип ×среда для всех количественных признаков, кроме ширины бобов. Фактор среды оказывает наибольшее влияние на фенотипическое проявление признаков, далее следуют факторы «генотип» и «генотип ×среда». По признакам «высота растения» и «высота бобов» образцы FbH 16 и FbH 13 можно отнести к высоким (79 и 35 см соответственно) и экологически стабильным (bi = 0.76, bi = 0.79). BGE 029055 имеет низкую вариабельность, с высокими значениями количества бобов (15) и количества семян с растения (41). Образцы $\mathrm{FbH}$ 14, FbH 16, FbH 15 и ВGP характеризуются высокой массой семян (от 28.36 до 34.93 г), но проявляют нестабильность (bi > 1) в неблагоприятных условиях среды. Fb 1903, BGE 043776 и Fb 3270, напротив, очень стабильны (bi < 1), однако низкопродуктивны. Промежуточные позиции занимают образцы Fb 1896, Fb 1929, Fb 2481, Fb 2486, BGE 002106 и BGE 029055, у которых коэффициент линейной регрессии приближается к единице, но они также являются низкопроизводительными. Интересен с позиций селекции образец BGE 041470, отличающийся высокими значениями массы 100 семян (101.38 г) 


\begin{abstract}
и массы семян с растения (32.14 г) и относительной стабильностью (bi = 1.10). Результаты анализа двойных диаграмм GGE позволяют расценивать образцы BGE 046721, BGE 032012, FbH 15 и FbH 16 как перспективный материал для селекции, сочетающий высокую и стабильную продуктивность семян.

Ключевые слова: Vicia faba L.; параметры стабильности; продуктивность; признаки; образцы; регрессионный анализ; непараметрический анализ.
\end{abstract}

\section{Introduction}

Broad bean (Vicia faba L.) is an important legume crop that is primarily used as a protein source in human diet, as forage for animals and as an available source of nitrogen for the biosphere (Rubiales, 2010). Enlargement of broad bean growing is desirable, but it is hampered due to unstable yields (Link et al., 2010).

Yield is a complex quantitative characteristic that is usually controlled by several genes and affected by environmental conditions. The importance of genotype-environment interaction in national programs for cultivar evaluation and breeding programs has been found in most major crops (Kendal et al., 2016; Pouresmael et al., 2018). Researchers investigate and describe stable genotypes by use of different parametric and nonparametric methods or single-variate and multivariate statistical methods (Hamayoon et al., 2011; Imtiaz et al., 2013; Kendal, Sayar, 2016).

In recent years, breeders' interest in legume crops, and especially in broad beans, has increased. Great attention is paid to the study of the starting material, both for the development of new varieties and for the improvement of the existing ones (Kurkina, 2013). In order to identify the best donors and promising candidate cultivars, statistical analysis of as many quantitative traits as possible can be used (Kurkina, 2003; Buravtseva et al., 2009).

The main direction in the breeding of legumes is the development of high-productive cultivars with stable yields over the years (Kazydub, Shamanin, 2003). The terms "plasticity" and "stability" are used to characterize the potential of modification variability and genotypic variability of the individual traits (or their groups). Plasticity (the ability of traits to change), as well as stability under different environmental conditions, are considered to be the basic adaptive properties of living organisms. Ecological plasticity of a genotype (cultivar) is the ability to a sustainable formation of high (compared to other genotypes) yield over a wide range of meteorological and agro-technical conditions (Zhuchenko, 2012; Marakaeva, Kazydub, 2016). Stability is a particularly important characteristic of cultivars in organic production conditions (Konvalina et al., 2009; Georgieva, 2017).

The aim of this study was to evaluate the ecological stability of broad bean accessions regarding main quantitative traits in broad bean in organic farming conditions.

\section{Material and methods}

The experimental activity was conducted at the Institute of Forage Crops (Pleven, Bulgaria) during the period 2016-2018. The objects of the study were 17 broad bean (Vicia faba L.) accessions of different origins: Portugal (Fb 1896, Fb 1903, Fb 1929, Fb 2481, Fb 2486, Fb 3270), Spain (BGE 002106, BGE 029055, BGE 032012, BGE 041470, BGE 043776, BGE 046721) and Bulgaria (FbH 13, FbH 14, FbH 15, FbH 16, BGP).The randomized block method (Barov, 1982) was used, with a plot size of $4 \mathrm{~m}^{2}$ and three replications. The sowing was done by hand, at a depth of $8 \mathrm{~cm}$ and with a rate of 30 seeds per $\mathrm{m}^{2}$. Plants were grown under organic farming conditions without the use of fertilizers and pesticides. A biometric estimation was performed, including the following traits: plant height $(\mathrm{cm}), 1$ st pod height $(\mathrm{cm})$, pods number per plant, pod lenght $(\mathrm{cm})$, pod width $(\mathrm{cm})$, seeds number per plant, seed weight per plant $(\mathrm{g}), 100$ seeds mass $(\mathrm{g})$.

The obtained data were processed by two-factor analysis of variance for each trait for determine of the influence of genotypes (accessions) $(\mathrm{G})$, environments (E) and their interaction $(G \times E)$. The evaluation of the ecological stability of the studied accessions was performed using the following analyzes and parameters: regression analysis - according to (Eberhart, Russell, 1966) where the regression coefficient (bi) and the variance of the deviations from regression $\left(\mathrm{Si}^{2}\right)$ were calculated; according to (Tai, 1979) in which the parameters $a_{i}$ (linear response to the environmental effects) and $\lambda_{\mathrm{i}}$ (deviation from the linear response) were determined; variance analysis - mean variance component (PP) according to (Plaisted, Peterson, 1979), ecovalence (W2) according to (Wricke, 1965), stability variances $\left(\sigma^{2}\right.$ and $\left.\mathrm{W}_{\mathrm{i}}\right)$ according to (Shukla, 1972) and (Annicchiarico, 1992) respectively; and non-parametric analysis - by the rank-parameter $\left(\mathrm{Si}_{2}\right)$ on the model of (Huehn, 1990a, b). Plaisted and Peterson's (1979) mean variance component (PP) was a measure of a cultivar's contribution to the $\mathrm{G} \times \mathrm{E}$ interaction and was determined from a total through "pair-wise" analysis. According to Annicchiarico's method (1992), a reliability index $\left(\mathrm{W}_{\mathrm{i}}\right)$ was calculated which estimates the probability of a particular genotype (cultivar) to show a performance below the environmental average or below any standard used. GGE biplot model was done, which uses decomposition of the singular value of first two principal components (Yan, 2002). All experimental data were processed statistically with using the computer software GENES 2009.7.0 for Windows XP (Cruz, 2009).

\section{Results}

\section{Analysis of variance}

The results of the two-factor analysis of variance of the traits (Table 1) confirmed the differences in the environmental conditions during the experimental period. With regard to the trait of pod width, the variances of factors were insignificant, on the basis of which it can be considered that genotype differences between the accessions were not essential. For all other traits, the influence of the factors was statistically significant, and the environment had the greatest share. The effect of genotype was stronger pronounced than that of GE interaction for the 1 st pod height, pod length and 100 seeds mass. In terms of plant height, the GE interaction was more pronounced. Consequently, for improving this trait, further research will be needed. The statistical significance of the factors genotype, environment and their interactions for almost all traits was an objective prerequisite for determining the stability parameters. 
Table 1. Mean squares in analysis of variance of broad bean accessions regarding main quantitative traits

\begin{tabular}{|c|c|c|c|c|c|c|c|c|c|}
\hline $\begin{array}{l}\text { Source } \\
\text { of variation }\end{array}$ & $\mathrm{DF}$ & $\begin{array}{l}\text { Plant } \\
\text { height }\end{array}$ & $\begin{array}{l}\text { 1st pod } \\
\text { height }\end{array}$ & $\begin{array}{l}\text { Pods } \\
\text { number }\end{array}$ & $\begin{array}{l}\text { Seeds } \\
\text { number }\end{array}$ & $\begin{array}{l}\text { Pod } \\
\text { lenght }\end{array}$ & $\begin{array}{l}\text { Pod } \\
\text { width }\end{array}$ & $\begin{array}{l}100 \text { seeds } \\
\text { mass }\end{array}$ & $\begin{array}{l}\text { Seed } \\
\text { weight }\end{array}$ \\
\hline Environment & 2 & $12851.1^{*}$ & $690.94^{*}$ & $286.32^{*}$ & $2926.02^{*}$ & $113.83^{*}$ & 1.20 & $35356.36^{*}$ & $9731.29^{*}$ \\
\hline Genotype & 16 & $177.13^{*}$ & $147.74^{*}$ & $34.32^{*}$ & $289.38^{*}$ & $17.72^{*}$ & 0.24 & $2292.23^{*}$ & $310.92^{*}$ \\
\hline $\mathrm{G} \times \mathrm{E}$ & 32 & $182.05^{*}$ & $72.12^{*}$ & $23.55^{*}$ & $263.85^{*}$ & $3.18^{* * * *}$ & 0.08 & $709.42^{*}$ & $239.80^{*}$ \\
\hline Env/Gen & 34 & $927.29^{*}$ & $108.52^{*}$ & $39.012^{*}$ & $420.45^{*}$ & $9.69^{*}$ & 0.14 & $2747.48^{*}$ & $848.94^{*}$ \\
\hline Env/Gen 1 & 2 & $605.38^{*}$ & $92.03^{*}$ & $23.24^{*}$ & $58.24^{*}$ & 1.30 & 0.13 & $5874.38^{*}$ & $608.41^{*}$ \\
\hline Env/Gen 2 & 2 & $1122.58^{*}$ & $156.85^{*}$ & $32.18^{*}$ & $273.06^{*}$ & $2.65^{*}$ & 0.05 & $452.619^{*}$ & $343.72^{*}$ \\
\hline Env/Gen 3 & 2 & $732.47^{*}$ & $205.65^{*}$ & $23.94^{*}$ & $136.60^{*}$ & $6.28^{* *}$ & 0.10 & $3476.60^{*}$ & $446.80^{*}$ \\
\hline Env/Gen 4 & 2 & $1051.84^{*}$ & $283.73^{*}$ & $23.23^{*}$ & $149.73^{*}$ & 1.78 & 0.01 & $3253.31^{*}$ & $436.17^{*}$ \\
\hline Env/Gen 5 & 2 & $903.05^{*}$ & $109.54^{*}$ & $17.44^{*}$ & $70.98^{*}$ & $9.37^{* * *}$ & 0.16 & $4837.00^{*}$ & $831.81^{*}$ \\
\hline Env/Gen 6 & 2 & $3035.11^{*}$ & $516.04^{*}$ & $12.72^{*}$ & $419.25^{*}$ & $2.57^{*}$ & 0.04 & $597.64^{*}$ & $59.35^{*}$ \\
\hline Env/Gen 7 & 2 & $903.00^{* *}$ & $3.64^{* *}$ & $20.17^{*}$ & $600.13^{*}$ & $17.01^{*}$ & 0.19 & $225.16^{*}$ & $455.60^{*}$ \\
\hline Env/Gen 8 & 2 & $951.12^{* * *}$ & $9.35^{* * *}$ & $29.57^{*}$ & $610.76^{*}$ & 2.38 & 0.11 & $295.67^{*}$ & $563.18^{*}$ \\
\hline Env/Gen 9 & 2 & $1326.65^{*}$ & $81.75^{*}$ & $17.65^{*}$ & $376.83^{*}$ & $15.97^{*}$ & 0.18 & $2068.95^{*}$ & $694.54^{*}$ \\
\hline Env/Gen 10 & 2 & $1073.02^{*}$ & $83.75^{*}$ & $19.26^{*}$ & $266.96^{*}$ & $17.48^{*}$ & 0.05 & $1285.91^{*}$ & $794.75^{*}$ \\
\hline Env/Gen 11 & 2 & $1380.87^{*}$ & $70.74^{*}$ & $7.68^{* *}$ & $36.51^{*}$ & 1.40 & 0.43 & $1209.42^{*}$ & $93.13^{*}$ \\
\hline Env/Gen 12 & 2 & $700.98^{*}$ & $44.45^{*}$ & $5.21^{* * *}$ & $3.46^{* *}$ & $7.83^{* *}$ & 0.21 & $3452.99^{*}$ & $383.41^{*}$ \\
\hline Env/Gen 13 & 2 & $469.91 *$ & $47.73^{*}$ & $6.51^{* *}$ & $306.33^{*}$ & $14.00^{*}$ & 0.22 & $3273.78^{*}$ & $1014.93^{*}$ \\
\hline Env/Gen 14 & 2 & $660.35^{*}$ & $11.43^{* *}$ & $345.16^{*}$ & $2023.68^{*}$ & $17.02^{*}$ & 0.08 & $3474.64^{*}$ & $2971.63^{*}$ \\
\hline Env/Gen 15 & 2 & $142.95^{*}$ & $2.34^{*}$ & $20.34^{*}$ & $710.91^{*}$ & $14.30^{*}$ & 0.17 & $2949.75^{*}$ & $1652.84^{*}$ \\
\hline Env/Gen 16 & 2 & $477.29^{*}$ & $14.25^{*}$ & $24.62^{*}$ & $658.21^{*}$ & $16.51^{*}$ & 0.01 & $5105.18^{*}$ & $1505.32^{*}$ \\
\hline Env/Gen 17 & 2 & $227.38^{*}$ & $111.63^{*}$ & $34.21^{*}$ & $446.05^{*}$ & $16.83^{*}$ & 0.33 & $4874.15^{*}$ & $1576.42^{*}$ \\
\hline Total & 50 & 1 & & & & & & & \\
\hline
\end{tabular}

Note: Genotypes: 1, Fb1896; 2, Fb 1903; 3, Fb 1929; 4, Fb 2481; 5, Fb 2486; 6, Fb 3270; 7, BGE 002106; 8, BGE 029055; 9, BGE 032012; 10, BGE 041470; 11, BGE 043776; 12, BGE 046721; 13, FbH 13; 14, FbH 14; 15, FbH 15; 16, FbH 16; 17, BGP.

* Significant at $p<0.1 ;{ }^{* *} p<0.05 ;{ }^{* *} p<0.01$.

\section{Evaluation of stability}

The influence of environmental conditions on the trait formation was expressed by the values of the stability parameters calculated by the different methods (Table 2 and Table 3 ). Regarding the plant height, when comparing the accessions, it was found that the $\mathrm{FbH} 13$ and $\mathrm{FbH} 16 \quad(79 \mathrm{~cm})$ had the greatest height, followed by $\mathrm{Fb} 3270$ and BGE 043776 (78 cm) (Fig. 1). For the experimental conditions, BGP and $\mathrm{FbH} 15$ were the most stable. They were characterized by very low values of bi ( $\mathrm{bi}=0.24$; bi $=0.31)$, as in $\mathrm{FbH} \mathrm{15}$, the value of $\mathrm{Si}^{2}$ was considerably lower $\left(\mathrm{Si}^{2}=43.97\right)$.

The accessions Fb1896, Fb 1929, BGE 046721 and FbH 14 were distinguished by mean stability for bi $(\mathrm{bi}=0.86-0.95)$ and low variance. From the highest plant accessions, particular attention should be paid to $\mathrm{FbH} 16$ and $\mathrm{FbH} \mathrm{13,} \mathrm{which}$ were definitely stable $(\mathrm{bi}=0.76$; bi $=0.79$ ) and weakly affected by the environmental conditions, unlike $\mathrm{Fb} 3270$ and BGE 043776, whose plants were also high but they exhibited strong variability. According to the values of the regression coefficient, BGE 002106, BGE 029055, Fb 2481 and Fb 2486 were stable, with height below the average for the group of tested accessions, and BGE 032012 and BGE 041470 exhi- bited plasticity with respect to this trait due to its high value (bi $=1.18-1.32$ ).

The trait of 1st pod height had high values in $\mathrm{FbH} \mathrm{16,}$ $\mathrm{Fb} 2481$ and $\mathrm{FbH}$ 13, followed by $\mathrm{Fb} 1929$ and BGE 046721. According to stability data, $\mathrm{FbH} 16$ exhibited high stability (bi $\left.=0.06 ; \sigma^{2}=4.17 ; \mathrm{W}_{\mathrm{i}}=103.31\right)$, while $\mathrm{Fb} 2481$ and Fb 1929 (considering the coefficient of linear regression bi as well as the parameter PP) were characterized by very well-expressed responsiveness ( $\mathrm{bi}>1$; $\mathrm{PP}=33.65-36.85$ ). Similarly was the behavior of $\mathrm{Fb} 1896, \mathrm{Fb} 1903, \mathrm{Fb} 2486$, $\mathrm{Fb}$ 3270, BGE 032012, BGE 041470 and BGE 043776, but they set their 1st pods at a relatively lower height. Plants of $\mathrm{FbH} 13$ formed their first pods highly and, according to most stability parameters, showed very low variability.

The largest number (15) of pods per plant was obtained from accessions BGE 029055 and $\mathrm{FbH} 14$ (see Fig. 1). The following positions were occupied by Fb 2486, BGE 043776 , BGP (12 pods per plant). According to the stability data of the considered trait (see Table 2), all parameters defined FbH 14 as unstable. It is notable that accessions that formed a larger number of pods (11-15) were highly variable and responsive to improvement in growing conditions. An exception was 
Table 2. Parameters of phenotypic stability in broad bean accessions regarding main quantitative traits

\begin{tabular}{|c|c|c|c|c|c|c|c|c|}
\hline \multirow[t]{2}{*}{ Accessions } & bi & $\mathrm{Si}^{2}$ & $a_{i}$ & $\lambda_{\mathrm{i}}$ & \multirow{2}{*}{$\begin{array}{l}\sigma^{2} \\
\text { Shukla, } 1972\end{array}$} & \multirow{2}{*}{$\begin{array}{l}\text { PP } \\
\text { Plaisted, } \\
\text { Peterson, } 1979\end{array}$} & \multirow{2}{*}{ Wricke, 1965} & \multirow{2}{*}{$\begin{array}{l}\mathrm{W}_{\mathrm{i}} \\
\text { Annicchiarico } \\
1992\end{array}$} \\
\hline & \multicolumn{2}{|c|}{ Eberhart, Russell, 1966} & \multicolumn{2}{|c|}{ Tai, 1979} & & & & \\
\hline \multicolumn{9}{|c|}{ Plant height } \\
\hline Fb 1896 & $0.86^{* *}$ & $24.51^{* *}$ & 0.8669 & 39.6022 & 14.69 & 38.9799 & 101.3134 & 96.6814 \\
\hline Fb 1903 & 1.06 & $184.23^{* *}$ & 1.0578 & 294.1470 & 936.34 & 79.4805 & 558.7322 & 91.1648 \\
\hline Fb 1929 & 0.93 & $52.96^{* *}$ & 0.9291 & 84.9413 & 39.15 & 44.8390 & 167.4867 & 91.5588 \\
\hline Fb 2481 & 1.09 & $100.43^{* *}$ & 1.0916 & 160.6005 & 142.12 & 57.8982 & 314.9792 & 91.9984 \\
\hline Fb 2486 & 1.09 & $3.10^{* *}$ & 1.0899 & 5.4691 & 71.45 & 32.0026 & 22.5111 & 86.7744 \\
\hline $\mathrm{Fb} 3270$ & $1.94^{* *}$ & $116.75^{* *}$ & 1.9450 & 186.5475 & -8.33 & 180.6308 & 1701.1350 & 88.8600 \\
\hline BGE 002106 & 1.08 & $14.99^{* *}$ & 1.0789 & 24.4243 & 38.59 & 34.9145 & 55.3980 & 84.1335 \\
\hline BGE 029055 & $1.10^{*}$ & $14.60^{* *}$ & 1.1085 & 23.7940 & 351.60 & 35.5501 & 62.5767 & 90.7561 \\
\hline BGE 032012 & $1.32^{* *}$ & 0.74 & 1.3240 & 1.7015 & 47.84 & 44.3418 & 161.8715 & 93.4103 \\
\hline BGE 041470 & $1.18^{* *}$ & $8.91^{* *}$ & 1.1838 & 14.7143 & 54.03 & 36.9819 & 78.7482 & 90.5619 \\
\hline BGE 043776 & $1.35^{* *}$ & 0.27 & 1.3512 & 0.9337 & 245.44 & 46.6715 & 188.1837 & 102.2922 \\
\hline BGE 046721 & 0.95 & $12.36^{* *}$ & 0.9498 & 20.2371 & 205.40 & 33.7193 & 41.9000 & 97.6550 \\
\hline $\mathrm{FbH} 13$ & $0.76^{* *}$ & $20.34^{* *}$ & 0.7619 & 32.9499 & 46.44 & 43.0864 & 147.6932 & 104.3693 \\
\hline $\mathrm{FbH} 14$ & $0.90^{*}$ & $31.41^{* *}$ & 0.9003 & 50.6029 & 23.40 & 39.7729 & 110.2691 & 95.6382 \\
\hline $\mathrm{FbH} 15$ & $0.31^{* *}$ & $43.97^{* *}$ & 0.3181 & 70.6230 & 19.58 & 104.0226 & 835.9135 & 88.7764 \\
\hline $\mathrm{FbH} 16$ & $0.79^{* *}$ & 0.03 & 0.7946 & 0.0476 & 118.85 & 35.6631 & 63.8529 & 105.7174 \\
\hline BGP & $0.24^{* *}$ & $119.96^{* *}$ & 0.2491 & 191.7374 & 0.33 & 137.4324 & 1213.2480 & 90.0438 \\
\hline \multicolumn{9}{|c|}{ 1st pod height } \\
\hline Fb 1896 & 1.29 & $15.77^{* *}$ & 1.2928 & 25.6599 & 24.01 & 16.5806 & 55.2583 & 86.9871 \\
\hline Fb 1903 & $1.92^{* *}$ & $4.24^{* *}$ & 1.9224 & 7.2371 & 186.74 & 19.0091 & 82.6858 & 89.5109 \\
\hline Fb 1929 & $1.50^{*}$ & $75.50^{* *}$ & 1.5045 & 120.8449 & 203.76 & 33.6578 & 248.1301 & 97.6634 \\
\hline $\mathrm{Fb} 2481$ & $2.24^{* *}$ & $52.64^{* *}$ & 2.2437 & 84.3306 & 25.86 & 36.8590 & 284.2840 & 100.6840 \\
\hline Fb 2486 & $1.58^{* *}$ & $4.93^{* *}$ & 1.5823 & 8.3652 & 32.82 & 15.5190 & 43.2677 & 72.4388 \\
\hline $\mathrm{Fb} 3270$ & $3.25^{* *}$ & $57.62^{* *}$ & 3.2526 & 92.0538 & 7.25 & 63.4964 & 585.1307 & 83.5793 \\
\hline BGE 002106 & $0.23^{* *}$ & 0.69 & 0.2262 & 1.5986 & 70.32 & 16.2578 & 51.6120 & 81.6485 \\
\hline BGE 029055 & $0.26^{* *}$ & $4.01^{* *}$ & 0.2658 & 6.8280 & 110.24 & 24.3403 & 142.8964 & 63.9726 \\
\hline BGE 032012 & 1.22 & $13.71^{* *}$ & 1.2223 & 22.3807 & 47.56 & 15.7731 & 46.1379 & 95.1409 \\
\hline BGE 041470 & 1.26 & $12.67^{* *}$ & 1.2577 & 20.7200 & 6.71 & 15.6185 & 44.3912 & 76.0487 \\
\hline BGE 043776 & 1.32 & 0.33 & 1.3196 & 0.0067 & 0.67 & 12.4233 & 8.3049 & 91.5196 \\
\hline BGE 046721 & 0.78 & $12.80^{* *}$ & 0.7802 & 20.9221 & 3.97 & 15.5222 & 43.3041 & 105.1743 \\
\hline $\mathrm{FbH} 13$ & 1.08 & 0.32 & 1.0838 & 0.0164 & 161.84 & 11.7412 & 0.6005 & 115.3304 \\
\hline $\mathrm{FbH} 14$ & $0.18^{* *}$ & $6.39^{* *}$ & 0.1811 & 10.6762 & 13.94 & 18.2874 & 74.5346 & 101.0185 \\
\hline $\mathrm{FbH} 15$ & 0.23 & 0.23 & 0.2309 & 0.1270 & 36.65 & 15.9604 & 48.2529 & 98.1128 \\
\hline $\mathrm{FbH} 16$ & $0.06^{* *}$ & 0.81 & 0.5578 & 1.6707 & 4.17 & 29.4062 & 200.1110 & 103.3181 \\
\hline BGP & $0.07^{* *}$ & $72.05^{* *}$ & 0.2763 & 115.2605 & 66.35 & 42.6060 & 349.1913 & 79.2728 \\
\hline \multicolumn{9}{|c|}{ Pods number } \\
\hline Fb 1896 & 1.13 & 0.74 & 1.1336 & 1.7139 & 11.73 & 3.9314 & 3.8258 & 99.3485 \\
\hline Fb 1903 & 0.58 & $17.32^{* *}$ & 0.5807 & 28.1234 & 28.86 & 8.8026 & 58.8420 & 70.7805 \\
\hline Fb 1929 & 1.19 & 0.21 & 1.1884 & 0.1928 & 28.67 & 3.7303 & 1.5543 & 67.0757 \\
\hline $\mathrm{Fb} 2481$ & 1.14 & 0.50 & 1.1430 & 1.3265 & 1.64 & 3.8745 & 3.1832 & 82.9719 \\
\hline Fb 2486 & 0.99 & 0.35 & 0.9874 & 1.0895 & 228.57 & 3.7748 & 2.0565 & 109.5617 \\
\hline Fb 3270 & $0.07^{* *}$ & $1.51^{*}$ & 0.7752 & 2.7405 & 14.35 & 13.4150 & 110.9343 & 72.4880 \\
\hline BGE 002106 & 0.98 & $2.30^{* *}$ & 0.9812 & 4.2002 & 21.31 & 4.2938 & 7.9188 & 84.8447 \\
\hline BGE 029055 & 1.27 & $1.19^{*}$ & 1.2739 & 2.4223 & 0.55 & 4.2193 & 7.0777 & 131.0650 \\
\hline
\end{tabular}


Table 2 (continued)

\begin{tabular}{|c|c|c|c|c|c|c|c|c|}
\hline \multirow[t]{2}{*}{ Accessions } & bi & $\mathrm{Si}^{2}$ & $a_{i}$ & $\lambda_{\mathrm{i}}$ & \multirow{2}{*}{ Shukla, 1972} & \multirow{2}{*}{$\begin{array}{l}\text { PP } \\
\text { Plaisted, } \\
\text { Peterson, } 1979\end{array}$} & \multirow{2}{*}{$\begin{array}{l}W^{2} \\
\text { Wricke, } 1965\end{array}$} & \multirow{2}{*}{$\begin{array}{l}W_{i} \\
\text { Annicchiarico, } \\
1992\end{array}$} \\
\hline & \multicolumn{2}{|c|}{ Eberhart, Russell, 1966} & \multicolumn{2}{|l|}{ Tai, 1979} & & & & \\
\hline BGE 032012 & 1.02 & 0.31 & 1.0226 & 0.0429 & 41.00 & 3.6013 & 0.0978 & 85.2169 \\
\hline BGE 041470 & 1.07 & 0.25 & 1.0662 & 0.1360 & 28.64 & 3.6284 & 0.4031 & 72.4842 \\
\hline BGE 043776 & 0.40 & $2.99^{* *}$ & 0.3974 & 5.2887 & 23.82 & 5.5536 & 22.1467 & 106.2217 \\
\hline BGE 046721 & 0.38 & 1.49 & 0.3816 & 2.8798 & 1.79 & 5.2091 & 18.2556 & 74.5156 \\
\hline $\mathrm{FbH} 13$ & 0.53 & 0.87 & 0.5271 & 1.9108 & 19.93 & 4.5757 & 11.1025 & 88.4313 \\
\hline $\mathrm{FbH} 14$ & $4.27^{* *}$ & $25.27^{* *}$ & 4.2791 & 40.1479 & 21.47 & 42.2413 & 436.5020 & 83.2290 \\
\hline FbH 15 & 0.77 & $6.54^{* *}$ & 0.7715 & 10.9442 & 28.78 & 5.5718 & 22.3528 & 82.5891 \\
\hline $\mathrm{FbH} 16$ & 0.95 & $5.89^{* *}$ & 0.9521 & 9.9328 & 0.15 & 5.2550 & 18.7748 & 86.7098 \\
\hline BGP & 1.09 & $9.17^{* *}$ & 1.0891 & 15.1406 & 17.43 & 6.1396 & 28.7652 & 99.9964 \\
\hline \multicolumn{9}{|c|}{ Seeds number } \\
\hline Fb 1896 & $0.33^{* *}$ & $26.11^{* *}$ & 0.3284 & 42.1215 & 42.46 & 64.4062 & 234.4991 & 78.2605 \\
\hline $\mathrm{Fb} 1903$ & $0.65^{* *}$ & $133.84^{* *}$ & 0.6458 & 213.8359 & 113.48 & 83.1058 & 445.6945 & 60.3994 \\
\hline Fb 1929 & 0.89 & 0.33 & 0.8908 & -0.0018 & 175.39 & 44.0063 & 4.1004 & 70.1788 \\
\hline Fb 2481 & 0.93 & 0.14 & 0.9318 & 0.3142 & 212.15 & 43.8375 & 2.1940 & 68.6338 \\
\hline $\mathrm{Fb} 2486$ & $0.23^{* *}$ & $41.06^{* *}$ & 0.2269 & 65.9392 & 737.04 & 72.8423 & 329.7770 & 93.4250 \\
\hline Fb 3270 & $0.31^{* *}$ & $83.03^{* *}$ & 0.3082 & 132.5242 & 248.78 & 228.0640 & 2082.8700 & 66.4390 \\
\hline BGE 002106 & $1.77^{* *}$ & $42.03^{* *}$ & 1.7659 & 67.4807 & 13.19 & 72.7638 & 328.8903 & 85.7961 \\
\hline BGE 029055 & $1.86^{* *}$ & $9.86^{* *}$ & 1.8603 & 16.2036 & 59.02 & 68.8939 & 285.1836 & 121.1575 \\
\hline BGE 032012 & $1.43^{* *}$ & $16.88^{* *}$ & 1.4282 & 27.4285 & 182.01 & 53.8015 & 114.7286 & 92.6802 \\
\hline BGE 041470 & 1.17 & $19.89^{* *}$ & 1.1726 & 32.2421 & 93.13 & 49.9243 & 70.9385 & 94.6878 \\
\hline BGE 043776 & $0.03^{* *}$ & $23.89^{* * *}$ & 0.0336 & 38.5313 & 83.77 & 82.6130 & 440.1294 & 66.6970 \\
\hline BGE 046721 & $0.13^{* *}$ & 0.08 & 0.1282 & 0.6190 & 708.10 & 66.9059 & 262.7313 & 92.4008 \\
\hline $\mathrm{FbH} 13$ & 0.92 & $106.55^{* *}$ & 0.9210 & 170.3375 & 317.61 & 72.2229 & 322.7817 & 74.6468 \\
\hline $\mathrm{FbH} 14$ & $2.86^{* *}$ & $415.14^{* *}$ & 2.8531 & 661.9547 & 327.45 & 258.6003 & 2427.7500 & 67.6624 \\
\hline $\mathrm{FbH} 15$ & $1.67^{* *}$ & $155.14^{* *}$ & 1.6662 & 247.7587 & 42.03 & 98.4585 & 619.0894 & 87.1802 \\
\hline $\mathrm{FbH} 16$ & $1.95^{* *}$ & $1.42^{*}$ & 1.9520 & 2.7444 & 123.01 & 71.7127 & 317.0196 & 62.5495 \\
\hline BGP & $1.57^{* *}$ & $14.03^{* *}$ & 1.5706 & 22.8795 & 218.19 & 57.3785 & 155.1271 & 126.6104 \\
\hline \multicolumn{9}{|c|}{ Pod length } \\
\hline Fb 1896 & 0.44 & 0.31 & 0.4310 & 0.0130 & 81.62 & 0.5798 & 4.3228 & 86.9333 \\
\hline $\mathrm{Fb} 1903$ & 0.59 & 0.12 & 0.5872 & 0.3292 & 467.37 & 0.4522 & 2.8821 & 93.9952 \\
\hline Fb 1929 & 0.82 & 0.84 & 0.8200 & 1.8710 & 81.77 & 0.5470 & 3.9528 & 89.4074 \\
\hline $\mathrm{Fb} 2481$ & 0.47 & 0.13 & 0.4660 & 0.3045 & 566.13 & 0.5830 & 4.3589 & 82.3804 \\
\hline $\mathrm{Fb} 2486$ & 1.05 & 1.01 & 1.0495 & 2.1340 & 722.30 & 0.5556 & 4.0493 & 92.5431 \\
\hline $\mathrm{Fb} 3270$ & 0.12 & $1.32^{*}$ & 0.1138 & 2.5855 & 212.64 & 1.5511 & 15.2931 & 62.7822 \\
\hline BGE 002106 & 1.59 & 0.21 & 1.5903 & 0.1739 & 38.76 & 0.6356 & 4.9527 & 94.2557 \\
\hline BGE 029055 & 0.56 & 0.12 & 0.5511 & 0.3297 & 641.46 & 0.4889 & 3.2962 & 80.6047 \\
\hline BGE 032012 & 1.52 & 0.02 & 1.5261 & 0.4861 & 10.04 & 0.6034 & 4.5894 & 104.2784 \\
\hline BGE 041470 & 1.55 & 0.67 & 1.5498 & 1.5747 & 261.03 & 0.8148 & 6.9767 & 129.3576 \\
\hline BGE 043776 & 0.43 & 0.23 & 0.4260 & 0.1475 & 112.51 & 0.6089 & 4.6519 & 77.4786 \\
\hline BGE 046721 & 0.84 & $1.74^{*}$ & 0.8391 & 3.2989 & 18.40 & 0.7774 & 6.5539 & 127.0036 \\
\hline $\mathrm{FbH} 13$ & 1.02 & $4.36^{* *}$ & 1.0200 & 7.4793 & 88.38 & 1.4441 & 14.0837 & 82.2417 \\
\hline $\mathrm{FbH} 14$ & 1.54 & 0.47 & 1.5422 & 1.2566 & 75.16 & 0.7519 & 6.2667 & 89.6194 \\
\hline $\mathrm{FbH} 15$ & 1.37 & 0.81 & 1.3744 & 1.8112 & 84.77 & 0.6636 & 5.2693 & 90.2566 \\
\hline $\mathrm{FbH} 16$ & 1.57 & 0.31 & 1.5738 & 0.0197 & 8.28 & 0.5873 & 4.4073 & 99.0947 \\
\hline BGP & 1.54 & 0.36 & 1.5402 & 1.0922 & 19.64 & 0.7221 & 5.9298 & 91.1550 \\
\hline
\end{tabular}


Table 2 (end)

\begin{tabular}{|c|c|c|c|c|c|c|c|c|}
\hline \multirow[t]{2}{*}{ Accessions } & bi & $\mathrm{Si}^{2}$ & $a_{i}$ & $\lambda_{\mathrm{i}}$ & \multirow{2}{*}{$\sigma^{2}$} & \multirow{2}{*}{$\begin{array}{l}\text { PP } \\
\text { Plaisted, } \\
\text { Peterson, } 1979\end{array}$} & \multirow{2}{*}{$\begin{array}{l}W^{2} \\
\text { Wricke, } 1965\end{array}$} & \multirow{2}{*}{$\begin{array}{l}\mathrm{W}_{\mathrm{i}} \\
\text { Annicchiarico } \\
1992\end{array}$} \\
\hline & \multicolumn{2}{|c|}{ Eberhart, Russell, 1966} & \multicolumn{2}{|l|}{ Tai, 1979} & & & & \\
\hline \multicolumn{9}{|c|}{100 seeds mass } \\
\hline Fb 1896 & $1.68^{* *}$ & $3.02^{* *}$ & 1.6800 & 5.2232 & 0.09 & 289.0503 & 1932.9440 & 112.0244 \\
\hline Fb 1903 & $0.47^{* *}$ & $4.59^{* *}$ & 0.4627 & 7.8220 & 6.34 & 225.5393 & 1215.6430 & 108.1333 \\
\hline Fb 1929 & $1.29^{* *}$ & 0.33 & 1.2930 & -0.0869 & 7.36 & 149.5031 & 356.8807 & 95.0492 \\
\hline Fb 2481 & $1.24^{* *}$ & $28.79^{* *}$ & 1.2423 & 46.3377 & 29.13 & 147.2577 & 331.5209 & 93.5870 \\
\hline $\mathrm{Fb} 2486$ & $1.35^{* *}$ & $685.76^{* *}$ & 1.3531 & 1093.4540 & -0.42 & 346.0680 & 2576.9080 & 90.3787 \\
\hline $\mathrm{Fb} 3270$ & $0.52^{* *}$ & $26.50^{* *}$ & 0.5177 & 42.8251 & 1.50 & 210.7062 & 1048.1160 & 38.9100 \\
\hline BGE 002106 & $0.29^{* *}$ & $33.31^{* *}$ & 0.2898 & 53.5880 & 2.41 & 312.5915 & 2198.8200 & 78.9987 \\
\hline BGE 029055 & $0.27^{* *}$ & $99.07^{* *}$ & 0.2655 & 158.6011 & 10.40 & 343.0164 & 2542.4430 & 75.0066 \\
\hline BGE 032012 & 0.95 & $121.02^{* *}$ & 0.9525 & 193.4120 & 23.81 & 150.9705 & 373.4545 & 92.5282 \\
\hline BGE 041470 & $0.73^{* *}$ & $108.76^{* *}$ & 0.7346 & 173.9030 & 38.61 & 172.8278 & 620.3135 & 110.6985 \\
\hline BGE 043776 & $0.76^{* *}$ & $3.12^{* *}$ & 0.7609 & 5.5056 & 2.60 & 139.8718 & 248.1039 & 91.2702 \\
\hline BGE 046721 & $1.21^{* *}$ & $260.45^{* *}$ & 1.2134 & 415.5642 & 3.81 & 203.9377 & 971.6716 & 86.1608 \\
\hline $\mathrm{FbH} 13$ & $1.21^{* *}$ & $164.59^{* *}$ & 1.2063 & 262.8200 & -0.31 & 177.3867 & 671.8013 & 87.0916 \\
\hline $\mathrm{FbH} 14$ & $1.26^{* *}$ & $120.22^{* *}$ & 1.2585 & 192.1197 & -0.01 & 174.5284 & 639.5201 & 96.0861 \\
\hline $\mathrm{FbH} 15$ & $1.18^{* *}$ & $27.04^{* *}$ & 1.1827 & 43.5746 & 5.37 & 137.4564 & 220.8246 & 90.3847 \\
\hline $\mathrm{FbH} 16$ & $1.56^{* *}$ & $38.68^{* *}$ & 1.5578 & 62.0823 & 17.32 & 242.8314 & 1410.9410 & 99.7928 \\
\hline BGP & 1.03 & $1779.01^{* * *}$ & 1.0298 & 2835.8290 & 0.18 & 590.8681 & 5341.7100 & 60.5632 \\
\hline \multicolumn{9}{|c|}{ Seed weight } \\
\hline Fb 1896 & 0.96 & $50.19^{* *}$ & 0.9646 & 80.5250 & 0.01 & 62.1813 & 153.0098 & 99.9013 \\
\hline Fb 1903 & $0.45^{* *}$ & $151.91^{* *}$ & 0.4489 & 242.6463 & 0.19 & 119.8607 & 804.4483 & 77.7036 \\
\hline Fb 1929 & $0.88^{*}$ & 0.20 & 0.8832 & 0.2065 & 0.08 & 50.0498 & 15.9948 & 71.2676 \\
\hline Fb 2481 & $0.87^{*}$ & 3.43 & 0.8672 & 5.9933 & 0.02 & 51.4193 & 31.4623 & 68.6158 \\
\hline Fb 2486 & $0.84^{* *}$ & $286.87^{* *}$ & 0.8370 & 457.7392 & 0.01 & 127.6183 & 892.0627 & 97.1530 \\
\hline $\mathrm{Fb} 3270$ & $0.05^{* *}$ & $38.11^{* *}$ & 0.0542 & 61.1723 & 0.01 & 149.4923 & 1139.1100 & 30.5370 \\
\hline BGE 002106 & $0.87^{*}$ & $12.46^{* *}$ & 0.8732 & 20.3965 & 0.01 & 53.6636 & 56.8105 & 93.8915 \\
\hline BGE 029055 & 0.89 & $66.65^{* *}$ & 0.8991 & 106.7460 & 0.44 & 67.4573 & 212.5978 & 105.2589 \\
\hline BGE 032012 & 1.10 & 0.31 & 1.1015 & 0.0368 & 0.04 & 49.6832 & 11.8554 & 99.4171 \\
\hline BGE 041470 & $1.10^{*}$ & $55.42^{* *}$ & 1.1146 & 88.8561 & 0.20 & 64.7745 & 182.2975 & 112.5134 \\
\hline BGE 043776 & $0.30^{* *}$ & $27.19^{* *}$ & 0.3008 & 43.8128 & 0.01 & 105.4840 & 642.0755 & 61.6103 \\
\hline BGE 046721 & $0.77^{* * *}$ & $31.03^{* *}$ & 0.7666 & 49.9871 & 0.12 & 62.4878 & 156.4716 & 96.1664 \\
\hline $\mathrm{FbH} 13$ & $1.15^{*}$ & $170.29^{* *}$ & 1.1515 & 271.9284 & 0.05 & 96.2819 & 538.1455 & 63.2004 \\
\hline $\mathrm{FbH} 14$ & $2.10^{* *}$ & $301.70^{* *}$ & 2.0977 & 481.2721 & 0.34 & 250.9772 & 2285.2930 & 75.4486 \\
\hline $\mathrm{FbH} 15$ & $1.56^{* *}$ & $176.57^{* *}$ & 1.5569 & 281.9522 & 0.11 & 127.0596 & 885.7535 & 76.3140 \\
\hline $\mathrm{FbH} 16$ & $1.62^{* *}$ & 0.90 & 1.6207 & 1.9782 & 0.20 & 88.0090 & 444.7108 & 68.7624 \\
\hline BGP & $1.46^{* *}$ & $234.63^{* *}$ & 1.4623 & 374.4689 & 0.16 & 132.7076 & 949.5417 & 78.5726 \\
\hline
\end{tabular}

BGE 029055, in which most of the stability parameters characterized it as relatively stable.

With respect to the number of seeds per plant, BGE 029055 , BGP and $\mathrm{FbH} 14$ can be distinguished, which formed 3541 seeds per plant. As in the previous trait, and in this one, accessions with an increased number of seeds showed clearly pronounced instability. However, the values of the parameters $\mathrm{Si}^{2}$ and PP gave reason to consider that BGE 029055
$\left(\mathrm{Si}^{2}=9.85 ; \mathrm{PP}=68.89\right)$ and $\mathrm{BGP}\left(\mathrm{Si}^{2}=14.03 ; \mathrm{PP}=57.37\right)$ had a breeding value. They had a high adaptive ability, which in conditions above the averages, could provide them with a high realization of the number of seeds per plant. Accessions $\mathrm{Fb} 2486$ and BGE 046721 were determined as very stable, with the coefficient of linear regression significantly smaller than 1 , parameter $\mathrm{W}_{\mathrm{i}}$ close to 100 and number of seeds above the average for the group of tested accessions. Their low adap- 
Table 3. Rank-parameter $\left(\mathrm{Si}_{2}\right)$ of the studied traits according to the model of Huehn $(1990 \mathrm{a}, \mathrm{b})$

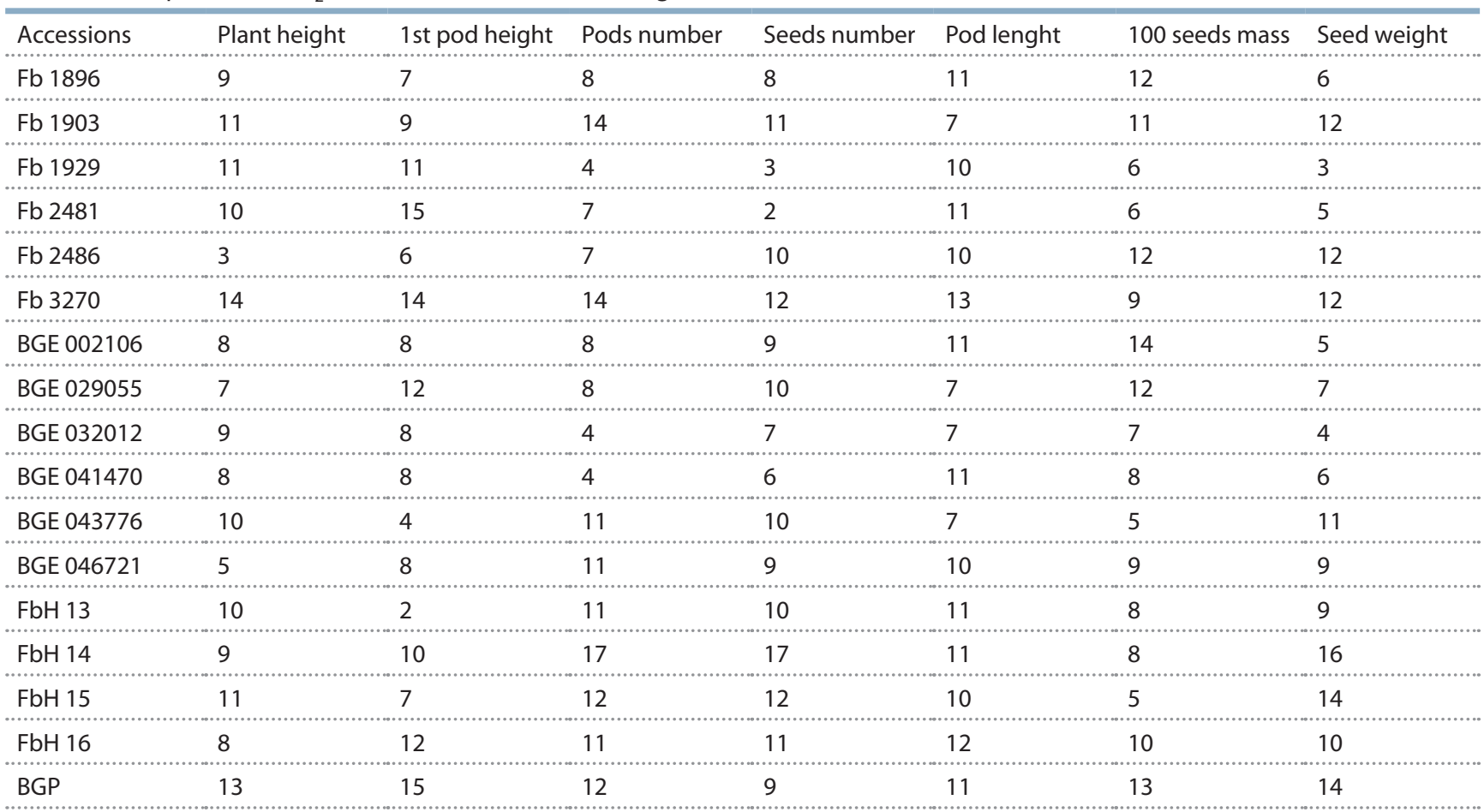

Note: $\mathrm{Si}_{2}=1$ : better performance; $\mathrm{Si}_{2}=17$ : worst performance.

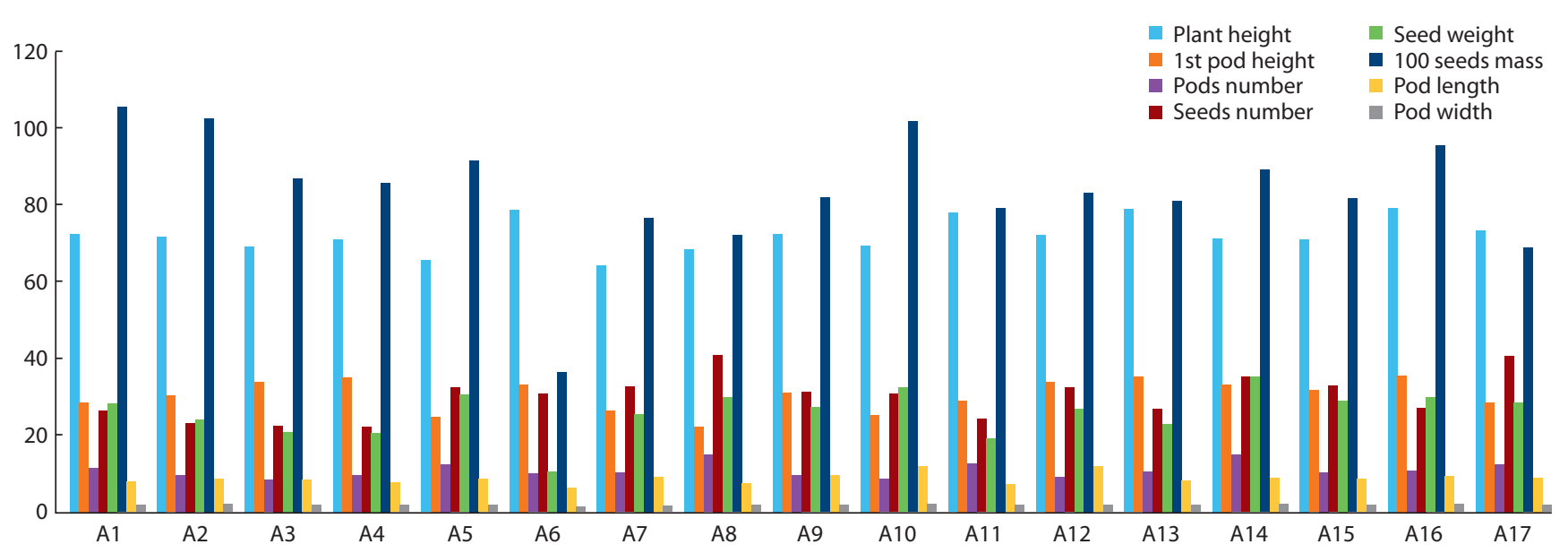

Fig. 1. Main quantitative traits in broad bean accessions (2016-2018).

Accessions: 1, Fb1896; 2, Fb1903; 3, Fb 1929; 4, Fb 2481; 5, Fb 2486; 6, Fb 3270; 7, BGE 002106; 8, BGE 029055; 9, BGE 032012; 10, BGE 041470; 11, BGE 043776; 12 , BGE $046721 ; 13, \mathrm{FbH} 13 ; 14, \mathrm{FbH} 14 ; 15, \mathrm{FbH} 15 ; 16, \mathrm{FbH} 16 ; 17, \mathrm{BGP}$.

tive ability implied that they were not suitable for growing at a high level of agro-technology, but they can exhibit well in unfavorable environmental conditions.

Because of the lack of significance in the stability parameters of Eberhart and Russell (1966) regarding the trait of pod length for all accessions, the complex evaluation of ecological stability was made on the basis of the remaining parameters. According to the parameter values based on variance analysis and nonparametric method of Huehn (1990a, b), $\mathrm{Fb} 1903$ and BGE 029055 outlined as the most stable, although they were one of the accessions with the shortest pods (see Table 3). A breeding compromise between the value of the trait and its stability (according to the indicator $\sigma^{2}$ ) can be found in BGE $046721\left(\sigma^{2}=18.4\right)$ and BGE $032012\left(\sigma^{2}=10.04\right)$. The ambiguous assessments according to the different types of parameters show that longer-term researches are needed to determine the environmental sustainability of this trait.

In terms of 100 seeds mass, Fb 1896 (105.48 g), Fb 1903 (102.34 g) and BGE 041470 (101.38 g) were clearly pronounced favorites, characterized by a high value of the trait (see Fig. 1). A comparison of the three types of stability parameters showed that $\mathrm{Fb} 1896$ was unstable and largely variable (see Table 2). Its reaction to the environment is highly predictable. It is suitable for growing in favorable environ- 
mental conditions. If conditions deteriorate, it can give way to the other accessions and to form seeds with smaller mass. Accession Fb 1903 also had a high mass of 100 seeds, but its coefficient of linear regression bi was less than 1 , so it can be defined as stable.

BGE 041470 was characterized by the fact that the coefficient bi (bi $=0.73$ ) was closer to 1 (compared to $\mathrm{Fb} 1903$ ), which determined it as closest to the "ideal" genotype for this trait. The stability parameters of Plaisted and Peterson (1979) and Annicchiarico (1992) confirmed the evaluation according to Eberhart and Russell (1966) and Tai (1979). This accession is of interest from a breeding point of view, combining a high mass of 100 seeds with ecological stability. It is suitable for growing in a wide range of environmental conditions.

Table 2 also presents data on the stability parameters of the seed weight per plant. FbH 14, FbH 16, Fb H 15 and BGP had high values of the regression coefficient ( $\mathrm{bi} \geq 1.5$ ), which determined them as unstable. With close average productivity (see Fig. 1) and similar values for bi (bi $=1.10-1.15$ ) were accessions FbH 13 and BGE 032012. More plastic, i.e. more responsive to favorable growing conditions was $\mathrm{FbH} 13$. Plants of BGE 041470 formed seeds with relatively large mass and were characterized by a coefficient of linear regression slightly above 1 . Because of these characteristics, this accession was of interest from a breeding point of view.

Accessions Fb 1896, Fb 1929, Fb 2481, Fb 2486, BGE 002106 and BGE 029055 had coefficients close to 1 , but they were distinguished by low productivity. Fb 1903 and BGE 043776 were stable under changing environmental conditions, with regression coefficient values of $\mathrm{bi}=0.45$ and $\mathrm{bi}=0.30$, respectively. In addition, BGE 043776 was also characterized by a low variance, i.e. its empirical values approximate to the theoretical ones. The most stable $(\mathrm{bi}=0.05)$, but with the lowest value of the considered trait was $\mathrm{Fb} 3270$.

\section{GGE biplot analysis}

The differences in stability of the accessions and their adaptability to the environment are presented in a two-dimensional coordinate system (Fig. 2). The axial axis shows the main effects (environment and genotype), and the ordinate axis the effects of their interaction (PC1 or PC2). On the vertices of the polygon are situated the accessions, which are furthest from the center. All accessions fall into this polygon. The lines separating the polygon of sectors represent a set of hypothetical environments. The accession, forming the polygon angle for each sector, has the highest value regarding the studied trait for the environment that falls within this sector.

In terms of the plant height, from the location of the accessions and environments (years), it is obvious that there is variability due to the influence of the environment and especially the climatic difference between the second (2017) and third (2018) experimental years. This result is in full compliance with the data from the variance analysis (see Table 1). The accessions, whose projections on the ordinate were close to the origin of the coordinate system, were stable and very weakly interact with the environment. These were $\mathrm{Fb} 1896$, Fb 3270, BGE 002106, Fb 1929, BGP, FbH 15. By contrast, accessions $\mathrm{Fb} 1903$ and BGE 043776 were the most distant therefore they were characterized by a specific reaction to the environment and low adaptation.
The result regarding the 1 st pod height indicated that the first two components (PC1 and PC2) determined $90.3 \%$ of the total variation of the trait as a result of GE interaction. The vertices of the polygon were occupied by Fb 1929, Fb 1903, BGE 002106, BGP, FbH 13, FbH 14 and FbH 15. Accessions $\mathrm{FbH}$ 16, BGE 043776 and BGE 002106 exhibited their biological potential the best during the third year. They were among the genotypes with the greatest environmental stability. Fb 2486, Fb 3270 and BGE 041470 developed relatively well in the first and second years. The location of the first year (closer to the center of the coordinate system) determined it as the most suitable environment for the broad bean development with respect to the considered trait.

The accessions, which were characterized by a number of pods above the average, had positive values of the first principal component $(\mathrm{PC} 1>0)$ and a value of $\mathrm{PC} 2$, tending to zero, were suitable for growing in different environments. In this trait, the accessions reacted differently to changing environmental conditions. In the most favorable position were those located in the quadrant, limited by the positive part of the abscissa (PC1) and the ordinate (PC2). From the accessions with a larger number of pods (FbH 14, BGP and BGE 029055), BGE 029055 can be preferred because of its lower variability. $\mathrm{Fb} 1903$ and $\mathrm{Fb} 3270$ were in the group of stable genotypes, however, they formed relatively few pods (10). A considerable part of the accessions had high (positive or negative) values of PC2, so they can be identified as having specific adaptability to the environment.

The graphical analysis of the stability in terms of the number of seeds per plant gave an analogous assessment obtained from the stability parameters. BGE 029055, BGP and FbH 14 had an increased number of seeds and occupied an extreme right position of the coordinate system, which determined them as the most productive and at the same time the most variable. Accessions Fb 1896 and Fb 1929 were one of the lowest productive, and their location close to the center of the coordinate system suggests a slight influence of the environment on the trait manifestation. In close proximity to them, with almost the same stability, were accessions Fb 2486 and BGE 046721. In breeding terms, they were more valuable because they formed a larger number of seeds.

GGE biplot analysis regarding 100 seeds mass showed that Fb 1929, BGE 032012 and BGE 043776 were located close to the origin of the coordinate system. They had low values on PC2 and PC1. This placement defined them as very stable, but they had a small mass of 100 seeds. BGE 041470 also had a small projection on PC2, but it was with a much higher value on the axial axis due to its larger mass of 100 seeds. Accessions Fb 1896 and Fb 1903 were distinguished with a high mass of 100 seeds and specific adaptability.

With regard to the trait of seed weight per plant, the first position was occupied by BGE 041470 , characterized by a very good combination between stability and level of the trait. Accessions Fb 2486, FbH 16, FbH 14, Fb 1896 and BGE 029055 were distinguished by relatively good productivity and stability, and can be recommended as suitable for growing conditions. The most pronounced variability of the considered trait had FbH 13 and $\mathrm{FbH} \mathrm{15,} \mathrm{and} \mathrm{BGE} 032012$ was characterized by the best stability but with very low productivity. 

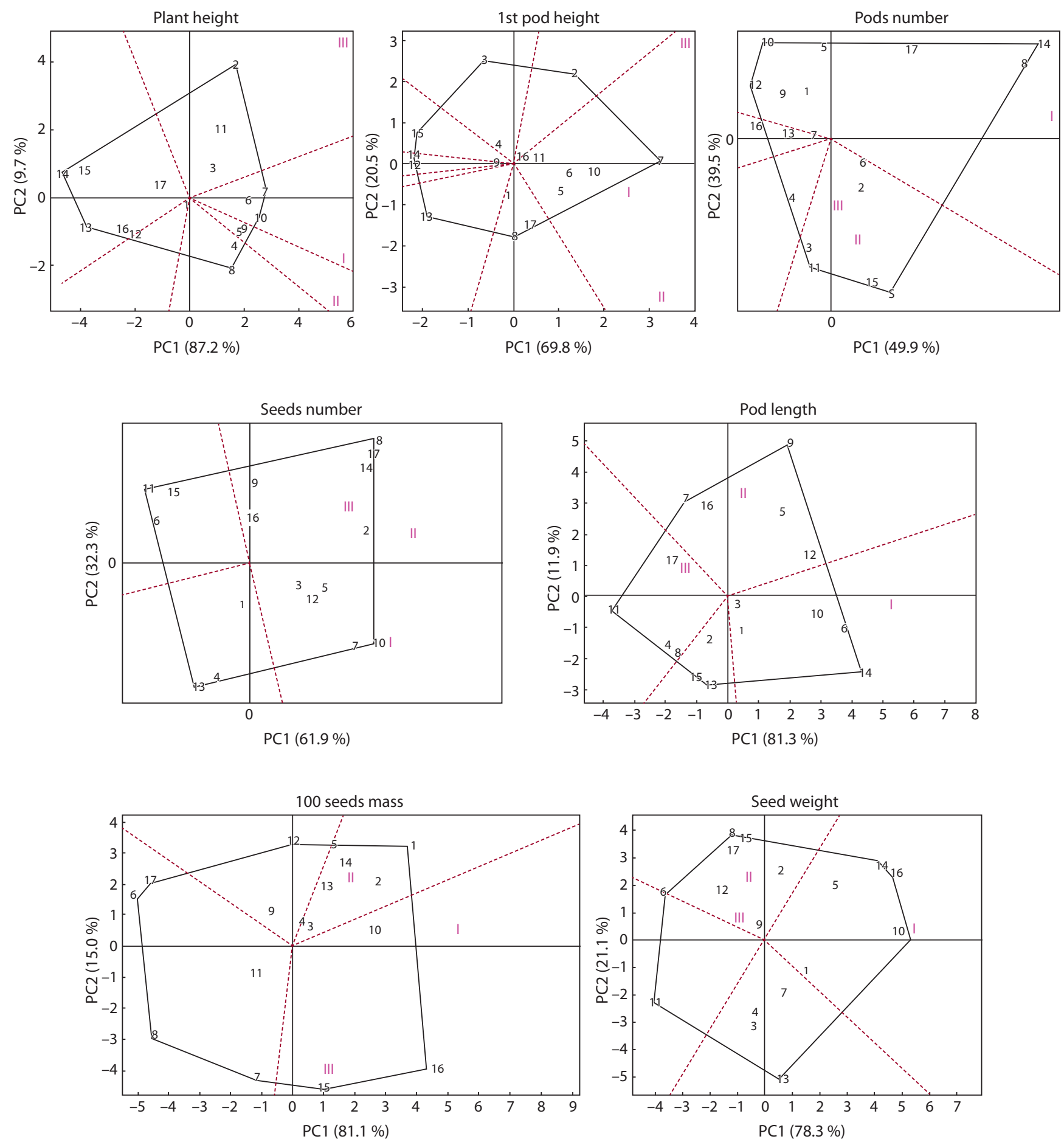

Fig. 2. GGE biplot analysis regarding the studied traits in broad bean accessions.

Accessions: 1, Fb1896; 2, Fb1903; 3, Fb 1929; 4, Fb 2481; 5, Fb 2486; 6, Fb 3270; 7, BGE 002106; 8, BGE 029055; 9, BGE 032012; 10, BGE 041470; 11, BGE 043776; 12 , BGE $046721 ; 13, \mathrm{FbH} 13 ; 14, \mathrm{FbH} 14 ; 15, \mathrm{FbH} 15 ; 16, \mathrm{FbH} 16 ; 17, \mathrm{BGP}$.

In Figure 3, $a$, the $\mathrm{X}$ axis (or the line of the average yield of the broad bean accessions) passes through the beginning of the coordinate system with an arrow indicating the positive end of the axis. The axis $\mathrm{Y}$ of the coordinate system (axis of stability) also passes through the beginning of the coordinate system perpendicular to the $\mathrm{X}$ axis. Thus, the average yield of the accessions can be estimated from the projection of their markers along the $\mathrm{X}$ axis, and the stability - from the projection along the $\mathrm{Y}$ axis. Accessions FbH 14, BGE 041470, Fb 2486 had the highest average yield, and Fb 3270, BGE 043776, $\mathrm{Fb} 2481$ and $\mathrm{Fb} 1929$ - the lowest one. The yield in FbH 14, BGE 041470, BGE 029055, Fb 1896 and Fb 1929 was the most variable, whereas $\mathrm{Fb}$ 2481, FbH 16 and BGE 046721 were distinguished with high stability.

The graphical analysis also enables to be determined accessions that combine high yield and stability. The center of 
$a$

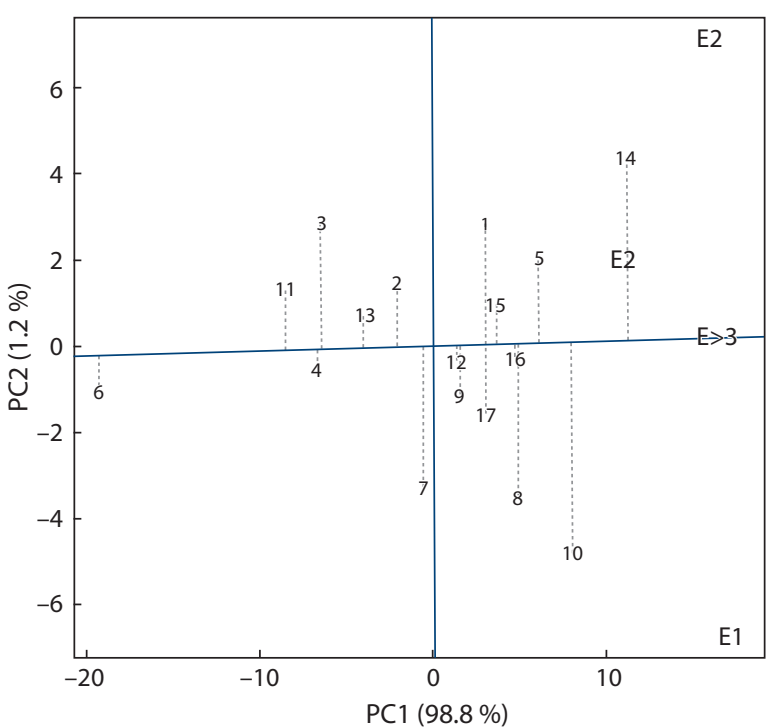

$b$

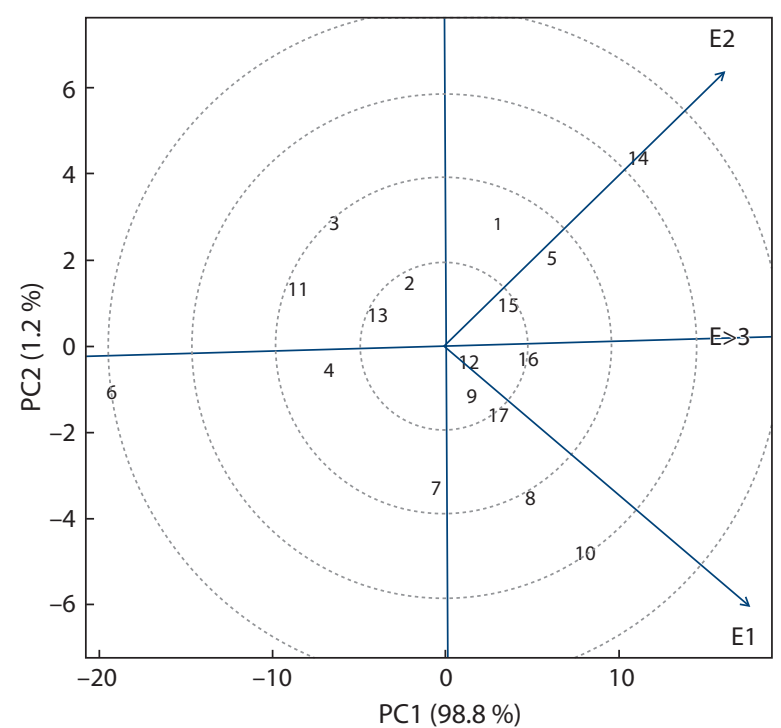

Fig. 3. GGE biplot analysis regarding seed yield in broad bean accessions.

Accessions: 1, Fb1896; 2, Fb1903; 3, Fb 1929; 4, Fb 2481; 5, Fb 2486; 6, Fb 3270; 7, BGE 002106; 8, BGE 029055; 9, BGE 032012; 10, BGE 041470; 11, BGE 043776; 12 , BGE 046721; 13, FbH 13; 14, FbH 14; 15, FbH 15; 16, FbH 16; 17, BGP.

Table 4. Correlation coefficients between stability parameters and seed weight per plant in broad bean accessions

\begin{tabular}{|c|c|c|c|c|c|c|c|c|c|}
\hline Parameter & bi & $\mathrm{Si}^{2}$ & $a_{i}$ & $\lambda_{\mathrm{i}}$ & $\sigma^{2}$ & PP & $W^{2}$ & $W_{i}$ & $\mathrm{Si}_{2}$ \\
\hline $\mathrm{Si}^{2}$ & 0.442 & & & & & & & & \\
\hline$a_{i}$ & $0.99^{* *}$ & 0.442 & & & & & & & \\
\hline$\lambda_{\mathrm{i}}$ & 0.442 & $0.99^{* *}$ & 0.442 & & & & & & \\
\hline$\sigma^{2}$ & 0.465 & 0.273 & 0.465 & 0.273 & & & & & \\
\hline PP & 0.348 & $0.769^{* *}$ & 0.348 & $0.769^{* *}$ & 0.309 & & & & \\
\hline$W^{2}$ & 0.348 & $0.769^{* *}$ & 0.348 & $0.769^{* *}$ & 0.309 & $0.99^{* *}$ & & & \\
\hline $\mathrm{W}_{\mathrm{i}}$ & 0.248 & 0.016 & 0.248 & 0.016 & 0.290 & -0.395 & -0.395 & & \\
\hline $\mathrm{Si}_{2}$ & 0.118 & -0.299 & 0.263 & $0.759^{* *}$ & 0.258 & $0.882^{* *}$ & $0.882^{* *}$ & -0.371 & \\
\hline Seed weight per plant & $0.530^{*}$ & 0.226 & $0.530^{*}$ & 0.226 & 0.369 & 0.111 & 0.111 & 0.335 & 0.162 \\
\hline
\end{tabular}

Note: bi, Si² - Eberhart and Russell (1966); $\mathrm{a}_{\mathrm{i}}, \lambda_{\mathrm{i}}$ - Tai (1979); $\sigma^{2}$ - Shukla (1972); PP - Plaisted and Peterson (1979); $\mathrm{W}^{2}$ - Wricke (1965); $W_{\mathrm{i}}-$ Annicchiarico (1992); $\mathrm{Si}_{2}-$ Huehn $(1990 \mathrm{a}, \mathrm{b})$. Significant at ${ }^{* *} p<0.01,{ }^{*} p<0.05$.

the concentric circles (Fig. 3, $b$ ) presents the position of the "ideal" genotype, which is defined by the projection of the environment axis determined by the longest vector of genotypes with yield above the average and lower projection of the perpendicular line (low variability in all environments). More preferred is this one, which is closer to the "ideal" genotype. In our study, BGE 046721, BGE 032012, FbH 13, Fb 1903, $\mathrm{FbH} 15$ and $\mathrm{FbH} 16$ were located closer to the center of the concentric circles, so they can be defined as close to the ideal type with regard to the magnitude and variability of seed yield compared to the rest accessions.

\section{Correlation analysis}

The results of the correlation dependences between seed weight per plant and the stability parameters were presented in Table 4 . The average productivity of the accessions positively, strongly and significant correlated with the parameters bi $(r=0.530)$ and $\mathrm{a}_{\mathrm{i}}(R=0.530)$. For the remaining parameters (especially $\mathrm{PP}, \mathrm{W}^{2}$ and $\mathrm{Si}^{2}$ ), the correlation was also positive but statistically insignificant and weaker.

The coefficient of regression bi interacted positively with the other parameters, especially with the parameter $\mathrm{a}_{\mathrm{i}}(r=$ $=0.990)$. The parameter $\mathrm{Si}^{2}$ (Eberhart, Russel, 1966) was in positive dependence with $\lambda_{\mathrm{i}}(r=0.99)$, PP $(r=0.769)$ and $\mathrm{W}^{2}(r=0.769)$. Strong positive correlations at a high level of statistical significance were found between $\mathrm{W}^{2}$ and the parameters $\lambda_{\mathrm{i}}(r=0.769)$ and $\mathrm{PP}(r=0.990)$.

The only negative correlations were established between the parameter $\mathrm{Si}_{2}$ (Huehn, 1990a, b) and $\mathrm{Si}^{2}$ (Eberhart, Russel, 1966) $(r=-0.299)$ and $\mathrm{W}_{\mathrm{i}}(r=-0.371)$, as well as between $\mathrm{W}_{\mathrm{i}}$ and respectively PP $(r=-0.395)$ and $\mathrm{W}^{2}(r=-0.395)$.

\section{Discussion}

According to (Ilchovska, 2008), for a more objective assessment of the genotype-environment interaction, the studied cultivars (genotypes) should be studied in a wider range of en- 
vironmental conditions. Some researchers (Yan, Tinker, 2006; Farshadfar et al., 2011) considered that in order to monitor the model of interaction between genotype and environment, and to better interpret the results, it is appropriate to use the biplot analysis. This polygonal graphic model represented the behavior and the interaction of genotypes in different environments, the differentiation of environment and also the specific environment for each of the genotypes studied.

The data obtained in this study regarding the interaction of genotype and environment confirmed the results of researches in other crops. In spring vetch, Sayar (2017) found that the factor 'environment' had the greatest impact on the yield, followed by the GE interaction and the factor 'genotype'. The presence of a considerable share of the factor 'environment' on the manifestation of main quantitative traits was reported by Tilahun et al. (2015) and Kanouni et al. (2015) in chickpeas, and by Sayar and Han (2016) in peas. Mortazavian et al. (2014) expressed an opinion that the stability analysis of the traits acquires important meaning when the effect of GE interaction is considerable.

The results of the correlation analysis of the data in our study were in support of the conclusions reached by other authors (Paula et al., 2014; Bornhofen et al., 2017) regarding the presence of positive relationships between the stability parameters and some main quantitative characteristics. According to Annicchiarico (2002), one of the possible explanations of this fact is that these methods (to determine the parameters mentioned) are related to the concept of static stability, which is obtained independently of the average expression of a given trait. The choice (made by these methods) may lead to the selection of stable but not necessarily productive genotypes.

Cargnelutti et al. (2009) concluded that the significant positive relationships between the parameters of the different methods showed that, in terms of stability, genotypes were evaluated in a similar way. The authors stated that the methods can provide much information and that only one method for stability evaluation would be sufficient to select the best genotypes. However, even in a strong relationship between the methods, the genotype position could be different in the different methods.

According to Balashova et al. (2013), one of the main tasks in the breeding of leguminous crops, including broad bean, was the seed yield stabilization. The authors considered that this problem can be solved by changing the idiotype of plants. According to them, one of the reasons for reducing the grain yield was the excessive increase in height and the lodging of plants. This was due to the fact that cultivars continue their vegetative growth after the formation of reproductive organs, especially in humid climatic conditions. For this reason, breeders' efforts should be directed to changing the plant habitus and development of highly productive determinant forms.

\section{Conclusions}

The results of the variance analysis in 17 broad bean accessions showed a significant genotype $\times$ environment interaction for all quantitative traits (excluding pod width). The factor environment had the greatest impact on the phenotypic manifestation of the traits, followed by the factors genotype and genotype $\times$ environment interaction.
In terms of plant height and 1st pod height, accessions $\mathrm{FbH} 16$ and $\mathrm{FbH} 13$ can be determined as high $(79 \mathrm{~cm}, 35 \mathrm{~cm})$ and ecologically stable $(\mathrm{bi}=0.76, \mathrm{bi}=0.79)$. BGE 029055 was low variable, with high values of the number of pods (15) and seeds (41) per plant.

Accessions FbH 14, FbH 16, FbH 15 and BGP were distinguished by high seed weight per plant (from 28.36 to $34.93 \mathrm{~g}$ ), but they exhibited instability ( $\mathrm{bi}>1$ ) under unfavorable environmental conditions. In contrast, Fb 1903, BGE 043776 and $\mathrm{Fb} 3270$ were very stable $(\mathrm{bi}<1)$ but low-productive. Intermediate positions occupied accessions $\mathrm{Fb} 1896, \mathrm{Fb} 1929$, $\mathrm{Fb} 2481, \mathrm{Fb} 2486$, BGE 002106 and BGE 029055, which had the coefficient of linear regression close to 1 , but they were also low-productive. Interesting from a breeding view point was BGE 041470 , which is characterized by high values of 100 seeds mass $(101.38 \mathrm{~g})$ and seed weight per plant $(32.14 \mathrm{~g})$, as well as with relative stability $(\mathrm{bi}=1.10)$.

GGE biplot analysis determined accessions BGE 046721, BGE 032012, FbH 15 and $\mathrm{FbH} 16$ as a promising breeding material combining high and stable seed yield.

\section{References}

Annicchiarico P. Cultivar adaptation and recomendation from alfafa trials in Northern Italy. J. Genet. Plant Breed. 1992;4:269-278.

Annicchiarico P. Genotype $\times$ environment interaction: challenges and opportunities for plant breeding and cultivar recommendation. FAO Plant Production and Protection Paper. Rome, 2002.

Balashova I.T., Pronina E.P., Sirota S.M., Gordeev D.K. Bean culture in Chernozem zone of Russia. Ovoschi Rossii = Vegetable Crops of Russia. 2013;1:60-62. (in Russian)

Barov V. Analysis and schemas of the field trial. Sofia, 1982. (in Bulgarian)

Bornhofen E., Benin G., Storck L., Woyann L.G., Duarte T., Stoco M.G. Statistical methods to study adaptability and stability of wheat genotypes. Bragantia. 2017;76(1):1-10.

Buravtseva T.V., Lagutina L.V., Gurkina M.V. Assessment of new beans source material from VIR collection and allocation of sources of economically important features. In: The Role of Genetic Resources and Breeding Achievements in Ensuring the Dynamic Development of Agricultural Production: Proc. of N. Int. Sci. Conf. (July 8-9, 2009, Orel). Orel, 2009;219-233.

Cargnelutti Filho A., Storck L., Riboldi J., Guadagnin J.P. Associação entre métodos de adaptabilidade e estabilidadeem em milho. Ciência Rural. 2009;39(2):340-347.

Cruz C.D. Programa Genes: Biometria. version 7.0. Viçosa, Brazil: Univ. of Federal Viçosa, 2009.

Eberhart S.A., Russel W.A. Stability parameters for comparing varieties. Crop Sci. 1966;6:36-40.

Farshadfar E., Zali H., Mohammadi R. Evaluation of phenotypic stability in chickpea genotypes using GGE-Biplot. Ann. Biol. Res. 2011; 2:282-292.

Georgieva N. Suitability of pea cultivars for organic farming conditions. Biol. Agric. Hortic. 2017;33(4):225-234.

Hamayoon R., Khan H., Naz S.L., Munir I., Arif M., Khalil I., Khan A. Performance of chickpea genotypes under two different environmental conditions. Afr. J. Biotechnol. 2011;10:1534-1544.

Huehn M. Non-parametric measures of phenotypic stability: Part 1. Theory. Euphytica. 1990a;47:189-194.

Huehn M. Non-parametric measures of phenotypic stability: Part 2. Application. Euphytica. 1990b;47:195-201.

Ilchovska M. Ecological assessment of common and special maize hybrids. In: Proc. of Int. Sci. Conf. of the Union of Scientists. Stara Zagora, 2008;56-62.

Imtiaz M., Malhotra R.S., Singh M., Arslan S. Identifying high yielding, stable chickpea genotypes for spring sowing: specific adaptation 
to location and sowing seasons in the Mediterranean region. Crop Sci. 2013;53:1472-1480.

Kanouni H., Farayedi Y., Saeid A., Sabaghpour S.H. Stability analyses for seed yield of chickpea (Cicer arietinum L.) genotypes in the Western Cold Zone of Iran. J. Agric. Sci. 2015;7:219-230.

Kazydub N.G., Shamanin V.P. Course of Lectures on Selection and Genetics of Legumes (peas, soybean, common beans, vetch, legumes). Omsk: OmGAU Publ., 2003. (in Russian)

Kendal E., Sayar M.S. The stability of some spring triticale genotypes using biplot analysis. J. Anim. Plant Sci. 2016;26:754-765.

Kendal E., Sayar M.S., Tekdal S., Aktaş H., Karaman M. Assessment of the impact of ecological factors on yield and quality parameters in triticale using GGE biplot and AMMI analysis. Pak. J. Bot. 2016; 48:1903-1913.

Konvalina P., Stehno Z., Moudry J. The critical point of conventionally bread soft wheat varieties in organic farming systems. Agron. Res. 2009;7(2):801-810.

Kurkina Yu. Biological dependence among legumes and their selection value: PhDissertation. Voronezh, 2003. (in Russian)

Kurkina Yu. Breeding of broad bean for seed yield. Vavilovskii Zhurnal Genetiki i Selektsii = Vavilov Journal of Genetics and Breeding. 2013;16(4/2):922-925. (in Russian)

Link W., Balko C., Stoddard F.L. Winter hardiness in faba bean: physiology and breeding. Field Crops Res. 2010;115:297-307.

Marakaeva T.V., Kazydub N.G. Assessment of ecological plasticity and stability of samples of haricot of Western Siberia vegetable in the conditions of the southern forest-steppe. Int. Res. J. 2016;48(5):181184. (in Russian)

Mortazavian S.M.M., Nikkhah H.R., Hassani F.A., Sharif-Al-Hosseini M., Taheri M., Mahlooji M. GGE biplot and AMMI analysis of yield performance of barley genotypes across different environments in Iran. J. Agr. Sci. Tech. 2014;16:609-622.

Paula T.M., Marinho C.D., Souza V., Barbosa M.H.P., Peternelli L.A., Kimbeng C.A. Relationships between methods of variety adaptabi- lity and stability in sugarcane. Genet. Mol. Res. 2014;13(2):42164225.

Plaisted R.L., Peterson L.C. A technique for evaluating the ability of selection to yield consistently in different location and seasons. Am. Potato J. 1979;36:381-385.

Pouresmael M., Kanouni H., Hajihasani M., Astraki H., Mirakhorli A., Nasrollahi M., Mozaffari J. Stability of chickpea (Cicer arietinum L.) landraces in National Plant Gene Bank of Iran for drylands. J. Agr. Sci. Tech. 2018;20:387-400.

Rubiales D. Faba beans in sustainable agriculture. Field Crops Res. 2010;115:201-202.

Sayar M.S. Additive main effects and multiplicative interactions (AMMI) analysis for fresh forage yield in common vetch (Vicia sativa L.) genotypes. Agric. For. 2017;63:119-127.

Sayar M.S., Han Y. Forage yield performance of forage pea (Pisum sativum spp. arvense L.) genotypes and assessments using GGE biplot analysis. J. Agr. Sci. Tech. 2016;18:1621-1634.

Shukla G.K. Some aspects of partitioning genotype-environmental components of variability. Heredity. 1972;28:237-245.

Tai G.C.C. Analysis of genotype - environment interactions of potato yield. Crop Sci. 1979;19:434-438.

Tilahun G., Mekbib F., Fikre A., Eshete M. Genotype × environment interaction and stability analysis for yield and yield related traits of kabuli-type chickpea (Cicer arietinum L.) in Ethiopia. Afr. J. Biotechnol. 2015;14:1564-1575.

Wricke G. Zur berechnung der ökovalenz bei sommerweizen und hafer. Pflanzenzuchtung. 1965;52:127-138.

Yan W. Singular-value partitioning in biplot analysis of multienvironment trial data. Agron. J. 2002;94:990-996.

Yan W., Tinker N.A. Biplot analysis of multi-environment trial data: principles and applications. Can. J. Plant Sci. 2006;86:623-645.

Zhuchenko A.A. Mobilization of Genetic Resources of Flowering Plants on the Basis of their Identification Systematization. Moscow, 2012. (in Russian)

\section{ORCID ID}

N. Georgieva orcid.org/0000-0002-6364-1310

V.I. Kosev orcid.org/0000-0003-3292-4200

Acknowledgements. The authors thank the Agricultural Academy in Bulgaria for funding the study.

Conflict of interest. The authors declare no conflict of interest.

Received March 20, 2019. Revised July 1, 2019. Accepted July 8, 2019. Published online August 30, 2019. 